\title{
Development of the Italian version of the Pain Catastrophising Scale (PCS-I): cross-cultural adaptation, factor analysis, reliability, validity and sensitivity to change
}

\author{
Marco Monticone $\cdot$ Paola Baiardi $\cdot$ Silvano Ferrari • \\ Calogero Foti · Raffaele Mugnai · Paolo Pillastrini • \\ Barbara Rocca $\cdot$ Carla Vanti
}

Accepted: 31 August 2011

(C) Springer Science+Business Media B.V. 2011

\begin{abstract}
Purpose The aim of this study was to create an Italian version of the Pain Catastrophising Scale (PCS-I) and evaluate its psychometric properties in a sample with chronic low back pain.

Methods The PCS was culturally adapted in accordance with international standards. The psychometric testing included factor analysis, reliability by internal consistency (Cronbach's alpha) and test-retest repeatability (intraclass coefficient correlations), and concurrent validity by comparing the PCS-I with a numerical rating scale (NRS), the Tampa Scale of Kinesiophobia (TSK), the Roland Morris Disability Questionnaire (RMDQ), the Hospital Anxiety and Depression Scale (HADS) and the Positive Affect and Negative Affect Scale (PANAS) (Pearson's correlation).
\end{abstract}

IRB approval. The Institutional Review Board approved the study, which was conducted in conformity with ethical and humane principles of research.

\section{Monticone ( $\square)$}

Physical Medicine and Rehabilitation Unit, Salvatore Maugeri Foundation, Institute of Care and Research (IRCCS),

Scientific Institute of Lissone, Via Monsignor Bernasconi 16, 20035 Lissone, MI, Italy

e-mail: marco.monticone@fsm.it

\section{P. Baiardi}

Scientific Institute of Pavia, Salvatore Maugeri Foundation

IRCCS and Consorzio Valutazioni Biologiche e

Farmacologiche, University of Pavia, Pavia, Italy

\section{S. Ferrari}

Manual Therapy Sciences, University of Padua, Padua, Italy

C. Foti

Chair of Physical and Rehabilitation Medicine,

Tor Vergata University of Rome, Rome, Italy
Results It took 4 months to develop an agreed version of the PCS-I, which was satisfactorily administered to 180 subjects with chronic low back pain. Factor analysis revealed a three-factor 13 -item solution (68\% of explained variance). The questionnaire was internally consistent with one exception ( $\alpha=0.92$ as a whole; 0.89 for Helplessness, 0.87 for Rumination and 0.56 for Magnification subscales) and showed a high degree of test-retest reliability $(\mathrm{ICC}=0.842)$. Concurrent validity showed moderate correlations with the NRS $(r=0.44)$, TSK $(r=0.59)$, RMDQ $(r=0.45$ ), HADS (Anxiety: $r=0.57$; Depression $r=0.46$ ) and PANAS (Negative Affect $r=0.54$ ). The minimum detectable change was 10.45 . The subscales were also psychometrically analysed.

Conclusion The successfully translated Italian version of the PCS has good psychometric properties replicating those of other versions.

\author{
R. Mugnai \\ Section of Occupational Medicine, Department of Internal \\ Medicine, Geriatrics and Nephrology, University of Bologna, \\ Bologna, Italy \\ P. Pillastrini \\ Section of Occupational Medicine, Department of Internal \\ Medicine, Geriatrics and Nephrology, University of Bologna, \\ Bologna, Italy \\ B. Rocca \\ Physical Medicine and Rehabilitation Unit, Salvatore Maugeri \\ Foundation, Institute of Care and Research (IRCCS), \\ Scientific Institute of Lissone, Milan, Italy \\ C. Vanti \\ School of Physiotherapy, University of Bologna, Bologna, Italy
}


Keywords Catastrophising - Low back pain · Validation . Assessment · Psychometrics

$\begin{array}{ll}\text { Abbreviations } \\ \text { LBP } & \text { Low back pain } \\ \text { PCS } & \text { Pain Catastrophising Scale } \\ \text { PCS-I } & \text { Pain Catastrophising Scale, Italian version } \\ \text { ICC } & \text { Intraclass coefficient correlation } \\ \text { NRS } & \text { Numerical rating scale } \\ \text { TSK } & \text { Tampa Scale of Kinesiophobia } \\ \text { RMDQ } & \text { Roland Morris Disability Questionnaire } \\ \text { HADS } & \text { Hospital Anxiety and Depression Scale } \\ \text { PANAS } & \text { Positive and Negative Affect Scale } \\ \text { MDC } & \text { Minimum detectable change } \\ \text { SEM } & \text { Standard error of the measurements } \\ \text { SD } & \text { Baseline standard deviation of the measurements } \\ \text { R } & \text { Test-retest reliability coefficient } \\ \text { r } & \text { Pearson's correlation }\end{array}$

\section{Introduction}

Low back pain (LBP) is a clinical condition that frequently affects adults [1]. According to the bio-psychosocial model, the transition from acute to chronic LBP and its persistence is due to the pathological interactions of individual, psychosocial and working factors [2]. Increasing evidence supports the crucial role of psychosocial factors, including psychological factors such as beliefs (e.g. catastrophising, fear of movement), everyday life strategies (e.g. coping), mood (e.g. anxiety, depression, distress), social factors (e.g. social support) and work (e.g. job satisfaction) which, by interacting with the environment, may lead to illness as they are the direct expression of an individual response to pain [3].

Catastrophising pain is one of the most important psychological variables explaining pain responses. It has been defined as 'an exaggerated negative orientation towards actual or anticipated pain experiences' and reflects a tendency to misinterpret or exaggerate apparently threatening situations [4] that can lead to increased sensitivity to pain, thus enmeshing patients in a vicious circle that may also involve reduced bodily performance.

To assess catastrophising more specifically, Sullivan developed the Pain Catastrophising Scale (PCS), a 13-item self-administered questionnaire consisting of three subscales called Rumination, Magnification and Helplessness [4]. Patients are asked to rate the degree to which they have any of the thoughts described in the questionnaire using a five-point scale. It has been shown that the PCS has a solid factor structure and satisfying psychometric properties, including internal consistency, test-retest stability and validity [4-7], and that it is related to physical and emotional health indices such as pain intensity, pain-related disability, pain-related fear and psychological distress [8, 9].

The PCS has been validated in German [10], Spanish [11], Catalan [12], Dutch [13], Chinese [14] and Singalese [15] and can therefore be used to make between-country comparisons. However, the absence of a psychometrically analysed Italian version has limited Italian researchers.

The aim of this paper is to describe the translation, cultural adaptation and validation of an Italian PCS in subjects with chronic LBP.

\section{Methods}

This descriptive study was approved by the Institutional Review Board of Salvatore Maugeri Foundation's Scientific Institute of Lissone (Italy). Patients gave their written consent to take part in the study.

Subjects

Outpatients referred to our rehabilitation hospital and three affiliated centres were enrolled between January and June 2010. The inclusion criteria were chronic common LBP, an age of $\geq 18$ years, and an ability to read and speak Italian fluently; the exclusion criteria were acute and subacute common LBP, specific causes of LBP, central or peripheral neurological signs, systemic illness or psychiatric deficits, and recent myocardial infarction, cerebrovascular events, or chronic lung or renal diseases.

The patients' demographic and clinical characteristics were recorded by a research assistant.

Translation and cross-cultural adaptation

The working group consisted of two medical doctors, one psychologist and another psychometrician.

The process of translation and cross-cultural adaptation of the PCS followed Beaton's guidelines [16] and involved translating it into Italian (two native Italian speakers compared their versions while keeping the language compatible with a reading age of 14 years), back-translating it into English (done by two bilingual mother-tongue English translators who were careful to reflect the same item content as the original), a review of the final version by a bilingual committee of clinicians and psychometric experts and testing the pre-final version (30 patients were asked what was meant by each item and the chosen response in order to verify whether the formulation of the items was clear). The PCS-I is reproduced in the appendix included as electronic supplementary material. 
Psychometric scale properties

\section{Acceptability}

The time taken to answer the questionnaire was recorded, the patients were asked about any difficulties that had been encountered, and all of the data were checked for missing or multiple responses.

\section{Factor structure}

The factor structure was analysed by means of a factor analysis, and subscales were stated. The exploratory factor analysis used Cattel's Scree Test to determine the number of extracted factors (eingenvalues of $>1$ ). Varimax rotation was applied, and the items with factor loadings of $>0.40$ were included in the factor.

\section{Reliability}

This was tested by means of internal consistency (Cronbach's alpha, with a value of $>0.70$ being considered acceptable) and day 1-7 test-retest stability (intraclass coefficient correlation-ICC) for the questionnaire as a whole and for each subscale.

\section{Validity}

Concurrent validity (i.e. the extent to which the scores relate to those of other measures in the expected manner) was calculated by comparing the PCS-I with a 0-10 numerical rating scale (NRS) [17], and the Italian versions of the Tampa Scale of Kinesiophobia (TSK) [18], the Roland Morris Disability Questionnaire (RMDQ) [19], the Hospital Anxiety and Depression Scale (HADS) [20], and the Positive and Negative Affect Scale (PANAS) [21]. We hypothesised that the PCS-I would measure aspects of the patient's complaints that were different from but related to those measured by the other instruments, which should lead to a moderate correlation. Pearson correlations: $r<0.30=$ fair; $0.30<r<0.60=$ moderate; $r>0.60=\operatorname{good}[22]$.

\section{Sensitivity to change [23]}

This was estimated using the minimum detectable change (MDC) calculated by multiplying the standard error of the measurements (SEM) by the z-score associated with the $95 \%$ confidence interval and the square root of 2 , which reflects the additional uncertainty introduced by using difference scores based on the measurements made at two time points (days 1 and 7). The SEM was estimated using the formula: $\mathrm{SEM}=\mathrm{SD}\left[(1-\mathrm{R})^{1 / 2}\right]$, where $\mathrm{SD}$ is the baseline standard deviation of the measurements, and $\mathrm{R}$ the test-retest reliability coefficient.

Statistical analyses were made using SPSS 16.0 (Italian version).

All of the participants were given written instructions concerning the questionnaires by a research assistant.

\section{Results}

Subjects

The study involved 180 subjects, 77 women (43\%) and 103 men $(57 \%)$ with a mean age of $44.1 \pm 11.3$ years (range 18-73). The median duration of LBP was 12 months (range 3-48). Table 1 shows their other socio-demographic characteristics.

Translation and cross-cultural adaptation

The process of forward/backward translation was undertaken by four translators. It took 2 months to reach a

Table 1 Socio-demographic characteristics of the population

\begin{tabular}{|c|c|c|}
\hline Variable & No & $\%$ \\
\hline \multicolumn{3}{|l|}{ Marital status } \\
\hline Unmarried & 74 & 41.1 \\
\hline Married & 106 & 58.9 \\
\hline \multicolumn{3}{|l|}{ Employment } \\
\hline Dependent employee & 115 & 63.9 \\
\hline Self-employed & 65 & 36.1 \\
\hline \multicolumn{3}{|l|}{ Education } \\
\hline Primary school & 10 & 5.6 \\
\hline Middle school & 20 & 11.1 \\
\hline High school & 77 & 42.7 \\
\hline University & 73 & 40.6 \\
\hline \multicolumn{3}{|l|}{ Smoking } \\
\hline Yes & 43 & 23.9 \\
\hline No & 137 & 76.1 \\
\hline \multicolumn{3}{|l|}{ Use of drugs } \\
\hline Antidepressants & 16 & 8.9 \\
\hline Analgesics & 74 & 41.2 \\
\hline Muscle relaxants & 20 & 11.1 \\
\hline NSAIDs & 70 & 38.8 \\
\hline \multicolumn{3}{|l|}{ Comorbidities (principal) } \\
\hline Hypertension & 57 & 31.6 \\
\hline NIDDM & 23 & 12.7 \\
\hline Heart disease & 10 & 5.6 \\
\hline Enteric disease & 11 & 6.1 \\
\hline Liver disease & 5 & 2.8 \\
\hline None & 74 & 41.2 \\
\hline
\end{tabular}


culturally adapted version; all of the items were easily translated and there were no difficulties in comprehension. A further review by experts and testing of the pre-final version (over a period of 2 months) confirmed the correctness of the translation/back-translation.

Psychometric scale properties

\section{Acceptability}

All of the questions were well accepted (100\%). The questionnaire was completed in $4.74 \pm 1.14 \mathrm{~min}$, without any missing responses or multiple answers. There were no problems in comprehension.

\section{Factor analysis}

Exploratory factor analysis revealed a three-factor structure on the basis of the number of eingenvalues of $>1$, which explained $68 \%$ of the variance. The three factors were Helplessness (items 2-6 and 12; factor loadings: 0.452-0.827), Rumination (items 1 and 8-11; factor loadings: 0.552-0.843) and Magnification (items 7 and 13; factor loadings: 0.544-0.886).

\section{Reliability}

Internal consistency: Cronbach's $\alpha$ index was 0.92 for the PCS-I as a whole ( 0.89 for Helplessness, 0.87 for Rumination and 0.56 for Magnification). Test-retest. Stability was measured in all of the subjects: the 1-7 day correlations were significant for the PCS-I as a whole (ICC = 0.842; 95\% CI: 0.766-0.890), as well as for the subscales (Helplessness: ICC $=0.858$; 95\% CI: 0.809-894; Rumination: $\mathrm{ICC}=0.831 ; 95 \%$ CI: $0.716-0.892$; Magnification: $\mathrm{ICC}=0.727 ; 95 \%$ CI: $0.649-0.790)$.

\section{Validity}

Table 2 shows the correlations between the PCS-I (including its subscales) and selected outcome measures: the PCS-I moderately correlated with the NRS $(r=0.44)$, TSK $(r=0.59)$, RMDQ $(r=0.45)$, HADS (Anxiety score: $r=0.57$; Depression Score $r=0.46$ ) and PANAS (Negative Affect $r=0.54$ ), and poorly correlated with the Positive Affect subscale of PANAS $(r=-0.32)$.

\section{Sensitivity to change}

The MDC was 10.45 , i.e., the smallest change in score likely to reflect a true change rather than a measurement error.

\section{Discussion}

The cross-cultural adaptation of the Italian PCS for chronic LBP guaranteed that the meaning of the original items was adequately captured by the idiomatic translation. The questionnaire proved to be highly acceptable and easily understood and could be self-administered in about $5 \mathrm{~min}$.

Factor analysis showed that the best fit was a threefactor 13-item model, as originally proposed [4] and subsequently confirmed by most of the other translations $[5,6$, 10-12, 14]. The item distribution of the original version was generally confirmed, the only exceptions being item 1 (loaded better on Rumination than on Helplessness) and item 6 (loaded better on Helplessness than on Magnification). These discrepancies were discussed by the experts and were accepted with respect to factor loadings and to clinical explanations: item 1 was interpreted as suggesting mulling over a thought rather than merely expressing Helplessness and therefore it was included in Rumination; item 6 suggested a lack of initiative rather than the exaggeration of pain and hence it was included in Helplessness.

Internal consistency of the scale as a whole was high $(\alpha=0.92)$, in line with the original $(0.87)$ and the translations into Dutch (0.85), Catalan (0.91), German (0.94), Chinese (0.92) and Singalese (0.89); the $\alpha$-value of the Spanish version was lower (0.79). As in previous studies $[4,10,12]$, satisfying estimates were reached for the Helplessness and Rumination subscales, but not by the Magnification subscale, probably because of its few items
Table 2 Validity: Pearson's correlations between PCS-I (and its subscales) and NRS, TSK, RMDQ, HADS and PANAS

All $P$ values $<0.001$

\begin{tabular}{lllll}
\hline Outcome measures & PCS-I & PCS-I helplessness & PCS-I rumination & PCS-I magnification \\
\hline NRS & $r=0.44$ & $r=0.38$ & $r=0.45$ & $r=0.28$ \\
TSK & $r=0.59$ & $r=0.52$ & $r=0.55$ & $r=0.46$ \\
RMDQ & $r=0.45$ & $r=0.49$ & $r=0.54$ & $r=0.36$ \\
HADS (anxiety score) & $r=0.57$ & $r=0.48$ & $r=0.52$ & $r=0.53$ \\
HADS (depression score) & $r=0.46$ & $r=0.38$ & $r=0.44$ & $r=0.39$ \\
PANAS (positive affect) & $r=-0.32$ & $r=-0.29$ & $r=-0.30$ & $r=-0.23$ \\
PANAS (negative affect) & $r=0.54$ & $r=0.44$ & $r=0.50$ & $r=0.52$ \\
\hline
\end{tabular}


[24]. It is therefore possible to question whether this subscale can be reliably used as an independent instrument.

The test-retest reliability of the PCS-I was highly significant (ICC $=0.842)$, higher than the English $(0.73)$ and Catalan versions (0.76), similar to the Spanish (0.84) and German versions (0.83), but lower than the versions in Dutch $(\alpha=0.92)$ and Chinese (0.96). However, our ICC estimates should be regarded with caution as they greatly depend on the between-subject variance of the enrolled sample.

As expected, the PCS-I showed moderate correlations with pain, fear of movement, disability and mood alterations, thus demonstrating that it measures a different but related concept that is unique during the multidimensional assessment of chronic LBP patients. As previously suggested [10], the fact that some correlations approached or exceeded 0.5 confirms redundancy among the measures [25].

Our results are largely in agreement with the original [4] and the other adapted versions [10-14]. Like Osman [6], we found a low correlation with positive affects, which suggests that catastrophisers are more likely to report low positive affect.

The PCS-I was also satisfactorily sensitive to change: at a $95 \%$ confidence level, the MDC indicates that a change of more than 10 points after a given intervention would not be a measurement error, a figure that is similar to that of the German adaptation (MDC $=12.8$ ) [10].

This study has some limitations. First of all, the PCS-I should have been validated using a 'gold standard' measure of catastrophising, but no other measure was available in Italian. Secondly, the relationships between self-reported beliefs and physical tests were not investigated as only selfadministered measures were used. Finally, since the study was restricted to patients with chronic LBP, it is uncertain whether the findings can be extended to other complaints of the locomotor system.

In conclusion, the PCS-I has good psychometric properties and can be recommended for the use in chronic LBP research in Italy.

Acknowledgments The authors would like to thank Francesca Bonetti, Teresa Coccia and Monia Rimondini for their assistance, and Kevin Smart for his help in preparing the English version of this paper.

\section{References}

1. Deyo, R. A., \& Weinstein, J. N. (2001). Low back pain. The New England Journal of Medicine, 344(5), 363-370.

2. Waddell, G., Newton, M., Henderson, I., Somerville, D., \& Main, C. J. (1993). A fear-avoidance beliefs questionnaire (FABQ) and the role of fear-avoidance beliefs in chronic low back pain and disability. Pain, 52(2), 157-168.

3. Pincus, T., Burton, A. K., Vogel, S., \& Field, A. P. (2002). A systematic review of psychological factors as predictors of chronicity/disability. Spine (Phila Pa 1976), 27(5), E109-E120.
4. Sullivan, M. J., Bishop, S. R., \& Pivik, J. (1995). The Pain Catastrophizing Scale: Development and validation. Psychological Assessment, 7, 524-532.

5. Van Damme, S., Crombez, G., Bijttebier, P., Goubert, L., \& Van Houdenhove, B. (2002). A confirmatory factor analysis of the Pain Catastrophizing Scale: invariant factor structure across clinical and non-clinical populations. Pain, 96, 319-324.

6. Osman, A., Barrios, F. X., Gutierrez, P. M., Kopper, B. A., Merrifield, T., \& Grittmann, L. (2000). The Pain Catastrophizing Scale: Further psychometric evaluation with adult samples. Journal of Behavioral Medicine, 23(4), 351-365.

7. Lamé, I. E., Peters, M. L., Kessels, A. G., Van Kleef, M., \& Patijn, J. (2008). Test-retest stability of the Pain Catastrophizing Scale and the Tampa scale for Kinesiophobia in chronic pain over a longer period of time. Journal of Health Psychology, 13, 820-826.

8. Severeijns, R., Vlaeyen, J. W. S., van den Hout, M. A., \& Weber, W. E. J. (2001). Pain Catastrophizing predicts pain intensity, disability, and psychological distress independent of the level of physical impairment. The Clinical Journal of Pain, 17, 165-172.

9. Picavet, H. S. J., Vlaeyen, J. W. S., \& Schouten, J. S. A. G. (2002). Pain Catastrophizing and Kinesiophobia: Predictors of chronic low back pain. American Journal of Epidemiology, 156, 1028-1034

10. Meyer, K., Sprott, H., \& Mannion, A. F. (2008). Cross-cultural adaptation, reliability, and validity of the German version of the Pain Catastrophizing Scale. Journal of Psychosomatic Research, 64, 469-478.

11. García Campayo, J., Rodero, B., Alda, M., Sobradiel, N., Montero, J., \& Moreno, S. (2008). Validation of the Spanish version of the Pain Catastrophizing Scale in fibromyalgia. Medicina Clínica (Barc), 131(13), 487-492.

12. Miró, J., Nieto, R., \& Huguet, A. (2008). The Catalan version of the Pain Catastrophizing Scale: A useful instrument to assess catastrophic thinking in whiplash patients. The Journal of Pain, 9(5), 397-406.

13. Crombez, G., Vlaeyen, J. W. S., Heuts, P. H. T. G., \& Lysens, R. (1999). Pain-related fear is more disabling than pain itself: Evidence on the role of pain-related fear in chronic low back pain disability. Pain, 80, 329-339.

14. Yap, J. C., Lau, J., Chen, P. P., Gin, T., Wong, Y., Phil, M., et al. (2008). Validation of the Chinese Pain Catastrophizing Scale (HK-PCS) in patients with chronic pain. Pain Medicine, 9(2), $186-195$

15. Pallegama, R. W., Ariyawardana, S. P. A. G., Ranasinghe, A. W., Sitheeque, M. A. M., \& Glaros, A. (2009). Validation of the Sinhala version of Pain Catastrophizing Scale. Proceedings of the Peradeniya University Research Sessions, 14, 94-96.

16. Beaton, D. E., Bombardier, C., Guillemin, F., \& Ferraz, M. B. (2000). Guidelines for the process of cross-cultural adaptation of self-report measures. Spine (Phila Pa 1976), 25, 3186-3189.

17. Huskinson, E. C. (1974). Measurement of pain. Lancet, 2, 1127-1131.

18. Monticone, M., Giorgi, I., Baiardi, P., Barbieri, M., Rocca, B., \& Bonezzi, C. (2010). Development of the Italian version of the Tampa Scale of Kinesiophobia, TSK-I. Cross-cultural adaptation, factor analysis, reliability and validity. Spine (Phila Pa 1976), 35(12), 1241-1246.

19. Padua, R., Padua, L., Ceccarelli, E., Romanini, E., Zanoli, G., Bondì, R., et al. (2002). Italian version of the Roland disability questionnaire, specific for low back pain: cross-cultural adaptation and validation. European Spine Journal, 11(2), 126-129.

20. Costantini, M., Musso, M., \& Viterbori, P. (1999). Detecting psychological distress in cancer patients: Validity of the Italian version of the hospital anxiety and depression scale. Supportive Care in Cancer, 7(3), 121-127. 
21. Watson, D., Clark, L. A., \& Tellegen, A. (1988). Development and validation of brief measures of positive and negative affect: the PANAS scales. Journal of Personality and Social Psychology, 54, 1063-1070.

22. Atkinson, G., \& Nevill, A. (1997). Comment on use of concordance correlation to assess the agreement between two variables. Biometrics, 52, 775-777.

23. Terwee, C. B., Bot, S., de Boer, M. R., van der Windt, D. A. W. M., Knol, D. L., Dekker, J., et al. (2007). Quality criteria were proposed for measurement properties of health status questionnaires. Journal of Clinical Epidemiology, 60, 34-42.
24. Osburn, H. G. (2000). Coefficient alpha and related internal consistency reliability coefficients. Psychological Methods, 5, 343-355.

25. Terwee, C. B., Van der Slikke, R. M., Van Lummel, R. C., Benink, R. J., Meijers, W. G., \& De Vet, H. C. (2006). Selfreported physical functioning was more influenced by pain than performance-based physical functioning in knee-osteoarthritis patients. Journal of Clinical Epidemiology, 59, 724-731. 